\title{
Actividades socioeconómicas en el Parque Nacional Isla del Coco, Costa Rica y posibles efectos de la variabilidad climática
}

\author{
Mary Luz Moreno-Díaz \\ Centro Internacional de Política Económica para el Desarrollo Sostenible, Universidad Nacional, Heredia, Costa Rica; \\ mmoreno@una.ac.cr
}

Recibido 09-III-2012. Corregido 03-VII-2012. Aceptado 01-X-2012.

\begin{abstract}
Socio-economic activities at Isla del Coco National Park, Costa Rica and possible effects of climate variability. Socioeconomic activities and their gross income in Isla del Coco National Park have been poorly evaluated and systematized, as well as the effects on them climate variability may have. A methodology that combines cluster analysis with a dynamic value chain was developed to identify the most important socioeconomic activities and quantify the gross income derived by the existence of Isla del Coco National Park. The possible effects of climate variability on these activities were also analyzed. A detailed literature review, consultation and interviews with researchers, entrepreneurs and tourists were conducted in order to apply the methodology. The main clusters of activities around this park are leisure and spiritual experiences, research and education, economic activities and park management. An approximation of the gross income from the activities carried out around the National Park in 2010 was calculated. The gross income generated at the national level was $\$ 5.7$ million dollars and at the international level was $\$ 2.5$ million dollars. The overall gross income was approximately $\$ 8.3$ million dollars. But, if the natural resources being visited by tourists degrade due to the effects of climate variability these benefits could decrease by 30\%. Citation: Moreno-Díaz, M.L. 2012. Actividades socioeconómicas desarrolladas alrededor y en el Parque Nacional Isla del Coco (Costa Rica) y posibles efectos del cambio climático sobre ellas. Rev. Biol. Trop. 60 (Suppl. 3): 113-129. Epub 2012 Dec 01.
\end{abstract}

Key words: Isla del Coco National Park, income, socioeconomic benefits, cluster, climate variability, added value, Costa Rica.

El Parque Nacional Isla del Coco (PNIC) fue declarado como tal en 1978, por el presidente Rodrigo Carazo Odio, mediante la emisión del Decreto Ejecutivo No. 8748-A, el cual fue ratificado posteriormente como Ley de la República, Ley No. 6794 del 09 de agosto de 1982. Cuenta con área terrestre de $24 \mathrm{~km}^{2}$, y con un área de ecosistemas marinos protegidos de $1997 \mathrm{~km}^{2}$ aproximadamente (Cajiao 2008). La Isla cuenta con varias bahías, puntas e islotes, así como arrecifes coralinos brindando refugio para las especies locales y migratorias, por ejemplo varios tipos de tiburones, rayas y cetáceos (Sibaja-Cordero 2008) que actualmente son objeto de visitación turística. Según Alfaro (2008) y Quirós-Badilla y Alfaro (2009) las variaciones estacionales en precipitación, viento, radiación, humedad relativa, temperatura superficial del aire y del mar, están asociadas a la migración meridional de la Zona de Convergencia Inter-Tropical, ya que la Isla del Coco se encuentra bajo su influencia prácticamente desde la primavera hasta el otoño boreal. Dicha zona está influenciada por el evento de El Niño-Oscilación Sur, que consiste en un calentamiento de las aguas en el Indo-Pacífico y cuyo calor es transportado por la ContraCorriente Ecuatorial hacia el Pacífico Oriental Tropical (Sibaja-Cordero 2008, Quirós-Badilla \& Alfaro 2009).

En el PNIC y sus alrededores se desarrollaron actividades humanas antes de su declaración como Parque Nacional en 1978. Hay evidencia de que por lo menos 28 expediciones 
fueron realizadas entre 1697 y esa fecha, las cuales tenían diferentes fines como la conquista, la exploración y científicos. A partir de 1978 se registran cerca de 20 expediciones científicas (Cortés 2008). Desde 1984, se permite la entrada al Parque a embarcaciones relacionadas con actividades ecoturísticas. Todas las actividades humanas que pueden desarrollarse en el PNIC están especificadas en el Plan de Manejo y Desarrollo y reguladas por el Decreto Ejecutivo No. 29537-MINAE del 20 de abril 2001 (Cajiao 2008). Entre estas actividades se contempla el ecoturismo, la investigación, el voluntariado y el trabajo de instituciones gubernamentales y no gubernamentales.

A pesar de la gran importancia socioeconómica y el ingreso que muchas de las Áreas Marinas Protegidas (AMP) generan a las actividades socioeconómicas que se desarrollan alrededor de ellas, existen pocos estudios que identifiquen dichas actividades y que cuantifiquen estos ingresos (Otoya et al. 2010). Con relación a otros aspectos que pueden impactar estas áreas como la variabilidad climática se han desarrollado algunos estudios físicos que evidencian los impactos pero no los cuantifica (Alvarado et al. 2011). Sibaja-Cordero (2008) realizó un análisis de 15 años de datos de avistamiento de poblaciones, recolectados por los guías de buzos en 27 puntos alrededor de Isla. Una de las conclusiones del estudio fue que el evento de El Niño ha tenido repercusiones sobre la abundancia y presencia de especies particulares, como el tiburón martillo (Sphyrna lewini) y la raya moteada (Taeniura meyeni), que disminuyen durante este fenómeno. El tiburón martillo presenta una estacionalidad aumentado en abundancia a partir de junio y descendiendo cerca de diciembre. El número de avistamientos cae abruptamente en años de El Niño (1992 y 1998). En el presente artículo se incluirán resultados obtenidos en el proceso de encuestación en los que se evidencia las posibles respuestas de los turistas a disminuciones en los recursos marinos turísticos como el tiburón martillo.

El objetivo del presente trabajo es identificar, caracterizar y cuantificar la generación de ingresos en actividades cuyo eje de acción es el PNIC. Además de realizar una aproximación de la posible disminución en esos ingresos debido a los efectos de la variabilidad climática sobre los recursos marinos de los que dependen estas actividades.

\section{MATERIALES Y MÉTODOS}

Conceptualización Metodológica. Los Parques Nacionales (PN) en general, y el PNIC en particular son activos naturales que generan servicios (ej: recreativos) aprovechados por conglomerados de actividades socioeconómicas para generar ingresos (Pearce \& Turner 1995, Furst et al. 2005). Estos servicios también son usufructuados por cadenas relacionadas de actividades conexas en escalas que trascienden a lo local debido a la existencia de impactos subsiguientes de tipo socioeconómico en la escala nacional e internacional, conduciendo potencialmente a un proceso de desarrollo vertical y horizontalmente vinculado (Moreno et al. 2011).

Aquí es relevante mencionar los conceptos de valor de uso y de no uso. Estos han sido trabajado por varios autores (Freeman 1993, Dixon et al. 1994, Garrod \& Willis 1999) y se han realizado aplicaciones en Areas Protegidas (Del Saz \& Suárez 1998, Chae et al. 2012, Wang \& Jia 2012). El valor de uso hace referencia al bienestar derivado del uso directo o indirecto de un bien y el de no uso al bienestar generado por la mera existencia del bien. En el caso de las Areas Protegidas el valor de uso se considera como aquel que obtienen los usuarios de la existencia de estas zonas como oferente de una serie de servicios, como son los de carácter recreativo, que afectan directamente al bienestar de estas personas. En el caso del PNIC el valor de uso se produce tanto para los proveedores de servicios de turismo al PNIC como para los visitantes que disfrutan de las bellezas naturales del mismo.

El instrumento que se percibe conceptual y operativamente como el más útil para realizar un análisis de las interrelaciones y externalidades positivas de un PN hacia las actividades 
económicas que giran en torno a él, es una combinación del análisis de conglomerado local y cadena transversal (es decir: desde la localidad de origen hasta el destino final).

El enfoque de conglomerado de actividades interrelacionadas dentro de una cadena de generación de ingreso, se identifica como la herramienta apta para la identificación de las actividades productivas (incluyendo proyectos de investigación en o relacionados con los parques y reservas) y reproductivas (en el sentido social e incluyendo la recreación de distinto nivel y calificación). Esto permite atribuir las contribuciones de $\operatorname{los} \mathrm{PN}$ a la creación y consolidación de dichas actividades de carácter socioeconómico para estimar por medio de cálculos numéricos y a través de indicaciones cualitativas los beneficios en forma de empleo, ingreso por visitación, entre otros (Fürst et al. 2004).

En el análisis del desarrollo socioeconómico, lo anterior se conoce como conglomerado vinculado a una dinámica de cadena de valor agregado (Porter 1990, 1999, Altenburg \& Meyer-Stahmer 1999, Ramos 1999). Para este artículo en particular, el conglomerado debe entenderse metodológicamente como una concentración sectorial y territorial de actividades y empresas que surgen a causa de determinadas necesidades (demanda por materias primas y servicios a suministrar por otras), que interactúan cada vez más entre ellas en términos productivo y organizacionales y que dependen en última instancia de la conservación o no del $\mathrm{PN}$ en estudio. Este enfoque permite identificar un amplio conjunto de efectos indirectos de encadenamiento y conexión (desde el punto de vista del cambio estructural del patrón de desarrollo socioeconómico) que a primera vista se concentran en un territorio alrededor del PN (en la llamada zona de influencia). Dicho impulso de desarrollo abarca también la emergencia de actividades de servicio y producción en espacios geográficos más allá de la zona de influencia directa. Involucra así unidades territoriales en la cadena de efectos originados por el PN, incluyendo la microregión, la región, el país y el entorno global que va desde lo local a la escala internacional.
La metodología plantea una enorme dificultad metodológica para identificar y delimitar, en términos socio-geográficos y análisis de cadena (eslabones económicamente y territorialmente encadenados), las diversas facetas de impacto inducidas en las escalas de impacto espacial indicadas. En particular, esto es válido para el traslape que se observa entre las escalas espaciales-territoriales (local, nacional e internacional) y el resultante problema de atribuir y contabilizar los ingresos a las unidades geográficas afectadas positivamente por la existencia y preservación del PNIC. El principal problema de atribución de ingresos al PNIC es identificar donde se genera el ingreso si en Puntarenas, en San Ramón, en San José o a nivel internacional. Por ejemplo la empresa de microbuses que lleva a los turistas a Puntarenas puede llenar su tanque en San José, en San Ramón o en Puntarenas. La compra de comida e insumos por parte de los Operadores Turísticos es otro ejemplo algunos se compran en Puntarenas y otros en San José.

Adicionalmente, el "doble conteo" de los ingresos asociados a las actividades desarrolladas dentro de las cadenas debe ser evitado con el objetivo de generar una cuantificación lo más precisa posible. El doble conteo, significa que un mismo monto de ingreso bruto se atribuye a dos actores en la misma cadena. Ejemplo de lo anterior, es que al considerar los ingresos brutos de los operadores turísticos que van al PNIC, se deben restar aquellos montos que ellos les pagan como ingreso a otros sectores tenidos en cuenta en el análisis como es el caso de las gasolineras.

Por lo que, el análisis procura evidenciar cualitativamente la forma cómo se organiza cada cadena, así como separar claramente qué ingresos se generan a partir de la existencia del PNIC y cuáles no. Estas limitaciones son tomadas en cuenta en el presente estudio en el momento de describir y cuantificar las contribuciones del parque.

Metodología específica. El presente trabajo se realizó durante los años 2010 y 2011. En una primera etapa se recopiló información 
secundaria (artículos, documentos de instituciones públicas) sobre las actividades socioeconómicas que se desarrollan en el PNIC. En la segunda etapa, se complementó esta información con entrevistas y encuestas a empresarios que desarrollan actividades alrededor de la existencia del PNIC y a turistas que lo visitan. Esta información se recopiló en forma personal, vía teléfono, fax y correo electrónico.

El trabajo de campo con los responsables de las actividades productivas se desarrolló durante el periodo de la investigación (20102011) y el realizado en el PNIC, incluyó dos expediciones del 19 al 29 de abril, 2010 y del 30 de junio al 10 de julio, 2011, en ellas se documentaron los atractivos turísticos del PNIC, la forma en que los empresarios realizan sus actividades turísticas en la zona y se realizaron entrevistas a los turistas, funcionarios encargados del PNIC y a voluntarios. En este trabajo de campo se realizaron encuestas a 126 turistas, nueve funcionarios del Área de Conservación Marina Isla del Coco (ACMIC) $\mathrm{y}$ del PNIC, tres funcionarios de actividades relacionadas (como guardacostas y voluntarios) y 11 a personas con actividades económicas. Adicional a lo anterior se entrevistaron 10 funcionarios de Organizaciones no Gubernamentales (ONG's) e instituciones públicas que desarrollan algún tipo de actividad en el PNIC. Es importante mencionar que en el año 2010 la Fundación Amigos del Coco (FAICO), organizó una expedición especial para turistas costarricenses en el barco Pacific Explorer, ese grupo fue encuestado para el presente artículo y por esto el número de costarricenses en la muestra para ese año puede ser más alto de lo que podría esperarse.

Una vez recolectada la información necesaria se procedió a realizar la caracterización de las actividades productivas, la identificación de los componentes del conglomerado de actividades desarrolladas alrededor del PNIC y de los encadenamientos respectivos, a la cuantificación de los ingresos obtenidos a nivel local, nacional e internacional y a analizar los posibles efectos de la variabilidad climática y el cambio climático sobre ellas. En términos de los ingresos a diferentes escalas, se identificó la ciudad de Puntarenas como el ámbito local, el resto del país como ámbito nacional y el resto del mundo como ámbito internacional.

En el presente artículo se realiza un análisis de toda la información obtenida y se calcula una aproximación a los aportes económicos de la existencia del PNIC para el 2010.

\section{RESULTADOS}

El análisis de conglomerados de actividades (Fig. 1), arrojó que los ejes principales alrededor de la protección y conservación del PNIC son: la recreación y la vivencia espiritual, la investigación y educación, la gestión y manejo y las actividades económicas.

\section{Recreación y vivencia espiritual}

Por medio de las actividades turísticas, los visitantes pueden entrar en contacto con la naturaleza; apreciando la belleza natural y conociendo la flora y fauna de la región. Esta es una contribución cualitativa muy importante que es la base del desarrollo de las actividades turísticas en el PNIC.

En el PNIC, el turista puede realizar una serie de prácticas recreativas específicas al Parque como buceo y caminatas entre otros. Dichas actividades dependen directamente de la conservación de los servicios ambientales como: la belleza escénica, la conservación de la biodiversidad marina y terrestre. Estas características atraen a miles de visitantes de todo el mundo.

Buceo: En esta actividad se pueden encontrar unas 270 especies de peces en su mayoría congregaciones de tiburones tales como: tiburón punta blanca (Charcharinus albimarginatus), martillo (Sphyrna lewini), punta negra (Carcharhimus limbatus) y ocasionalmente el tiburón ballena (Rhincodon Typus), siendo uno de los sitios de buceo más exigentes a nivel mundial, brindando a los visitantes un espectáculo único. Las zonas autorizadas para bucear son: Isla Manuelita, Isla Cónica, 


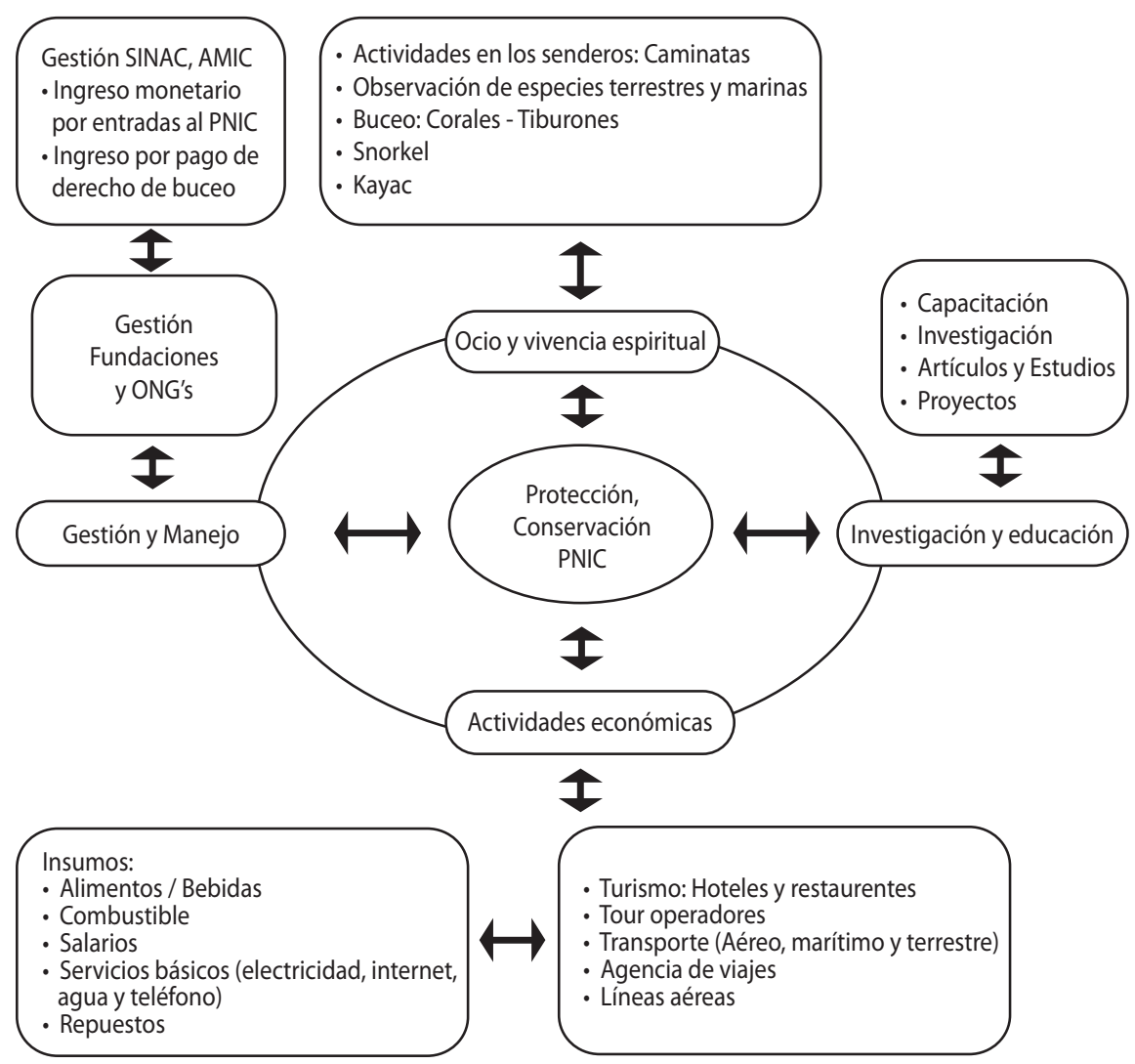

Fig. 1. Conglomerado de actividades desarrolladas alrededor del Parque Nacional Isla del Coco, Costa Rica.

Fig. 1. Cluster of activities developed around Isla del Coco National Park,Costa Rica.

Piedra Sucia, Isla Cáscara, Isla Pájaro, Punta Ulloa, Roca Sumergida, Cabo Dampier, Dos Amigos I y II, Aleta de Tiburón, Punta María y Bajo Alcyone. Para practicar esta actividad recreativa, se requiere supervisión profesional y equipamiento adecuado, por lo anterior los grupos de turistas van acompañados siempre de un especialista que es parte de la tripulación de las embarcaciones. El buceo nocturno es una práctica importante que permite al turista observar tiburones y otras especies marinas, debe desarrollarse una vez durante la noche, en grupos no mayores a 11 personas. (MINAESINAC-ACMIC 2007a).

Caminatas en los senderos: Para las actividades recreativas en la parte insular, se cuenta con tres senderos o caminos en los cuales el visitante puede realizar observación de flora, fauna. Estos senderos son: Chatham-Wafer, Cascada del Río Genio y Sendero al Cerro Yglesias (Fig. 2). La administración del Parque ha invertido esfuerzos en mejorar aquellos trayectos que puedan ser peligrosos para los turistas por caídas, por medio de programas como el de Ecoturismo (MINAE-SINAC-ACMIC 2007b).

Gestión y Manejo: Este eje está relacionado con el trabajo que realiza el Sistema Nacional de Áreas de Conservación (SINAC), en la protección y administración del PNIC y en las organizaciones que los apoyan como ONG's y fundaciones (MARVIVA, Fundación Amigos de la Isla del Coco -FAICO-, el 


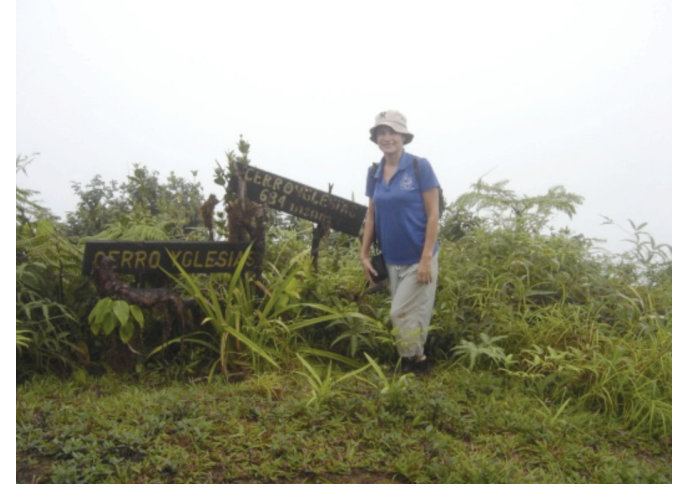

Fig. 2. Punto más alto para los visitantes en el Cerro Yglesias.

Fig. 2. Highest point for visitors in the CerroYglesias.

Programa Restauración de Tortugas Marinas -PRETOMA- entre otras). Este Parque Nacional se encuentra en la jurisdicción del Área de Conservación Marina Isla del Coco (ACMIC).

Dentro del ACMIC se desarrollan cinco programas de manejo del PNIC (BermúdezAcuña et al. 2007):

1. Programa de administración y operaciones: Desarrolla temas tales como: manejo financiero, recursos humanos, infraestructura, equipo, voluntariado, planificación, comunicación, seguimiento y evaluación, entre otros.

Dos de las organizaciones que han apoyado este programa son el Instituto Costarricense de Electricidad (ICE) y FAICO. Con la construcción de la microcentral hidroeléctrica Río Genio y de cabinas para los funcionarios respectivamente. El ICE inició la construcción de la microcentral hidroeléctrica Río Genio a inicios de 2002 y el 4 de febrero del 2005 se entrega oficialmente a los funcionarios del ACMIC (Obando 2005). La microcentral se creó con el objetivo de brindar energía eléctrica más limpia para cubrir las necesidades en la administración del PNIC y evitar algunos inconvenientes ocasionados por la generación térmica (ruido, traslado de combustible, contaminación, entre otras).

El ICE trabajó en conjunto con el ACMIC, que se constituye en el principal beneficiado del proyecto, colaborando en la construcción, hospedaje, alimentación, entre otros. Al ser un proyecto muy complejo, el ICE realizó sus obras con ayudas de algunas entidades externas, entre las que se pueden mencionar a FAICO que realizó una donación del equipo turbogenerador; el Servicio Nacional de Guardacostas que colabora con traslado de personal y materiales; algunos de los Operadores Turísticos también facilitaron el traslado de material y funcionarios; el gobierno de Japón donó las turbinas para la microplanta. Cabe resaltar que se le realizaron algunas modificaciones a la Planta Hidroeléctrica del Río Genio, la cual cuenta en la actualidad con una mejor infraestructura.

Por otro lado, FAICO gestionó fondos para la construcción de módulos habitacionales para los funcionarios del PNIC: dos módulos son de Holanda y uno de Japón, la empresa privada y recursos propios de la Fundación. La construcción en el sitio de estos módulos se llevó a cabo gracias al esfuerzo de grupos de voluntarios, funcionarios de FAICO y del PNIC.

2. Programa de financiamiento: Involucra temas de gestión de proyectos, mercadeo del parque, relaciones con Organizaciones no gubernamentales, entre otros.

En este componente el ACMIC es apoyado por organizaciones como FAICO que realiza distintas actividades y programas con el fin de adquirir fondos. Se tienen proyectos de alcancías en el aeropuerto, se hacen eventos de recaudación como las cenas de gala, degustación de vinos o el crucero a la Isla, se tienen programas de reciclaje y el programa de padrinos isla del coco. Estos proyectos permiten también 
sensibilizar a la población mostrando los distintos riesgos, dificultades y maravillas que existen en torno al PNIC (Villalobos com. pers. 2010)

3. Programa de control y vigilancia: temas de patrullaje, operativos especiales, control de áreas críticas, demarcación de límites y del equipo del parque, entre otros.

Los funcionarios del SINAC son apoyados por funcionarios de Guardacostas y la Fundación MARVIVA que en febrero del 2004 establece un convenio Marco de Cooperación con el MINAET para apoyar en la protección del PNIC. Otras instituciones como FAICO han donado embarcaciones empleadas para apoyar este componente.

4. Programa de manejo de recursos naturales y culturales: Abarca temas de investigación, monitoreo biológico, daño ambiental, recuperación de hábitats, manejo de especies introducidas, habilidad ecológica, entre otras.

Se destacan los aportes realizados por las universidades estatales y por ONG's como MARVIVA, FAICO y PRETOMA entre otras en este componente. Este programa brinda el sustento científico para la ampliación, diseño y manejo del PNIC. También, se trabaja con la población para brindar información sobre los recursos marinos.

5. Programa de uso público: involucra temas de uso sostenible, monitoreo de impactos por visita, interpretación ambiental, entre otros.

En este programa se destaca el apoyo que ha tenido el ACMIC por parte de Programa de Naciones Unidas para el Desarrollo (PNUD), El Fondo Mundial del Medio Ambiente (GEF), el Fondo Francés para el Medio Ambiente (FFEM) y Onca Natural $(\mathrm{ONG})$ para la elaboración del plan de uso y el de monitoreo de las zonas de uso público.

Dentro de los aportes socioeconómicos del PNIC (y del ACMIC) se destacan la generación de empleo y la atención de turistas que genera ingresos para el país.

El ACMIC, cuenta aproximadamente con 30 funcionarios, $75 \%$ hombres y $25 \%$ mujeres. Aproximadamente $19 \%$ de los funcionarios son procedentes de Puntarenas y $81 \%$ de otras zonas del país. La mayoría de los empleados poseen secundaria completa $(64 \%)$, otros licenciatura (21\%), bachillerato universitario $(12 \%)$ y un pequeño grupo cuenta con maestría (3\%). De acuerdo a los registros llevados por el ACMIC, en el año 2010 el monto por salarios para estos funcionarios fue de aproximadamente US $\$ 438,755.41$. El 68\% de estos fondos provienen directamente del SINAC y el 32\% del Fondo Nacional de Parques (FNP).

La visitación al PNIC para el 2010 fue de 3624 personas, de los cuales 1031 fueron exonerados del pago de la entrada (investigadores, científicos, entre otros). Teniendo en cuenta la tarifa de entrada (25US\$ por persona por día para extranjeros, nacionales y residentes), se obtiene que el total de ingresos percibidos por concepto de entradas y buceo al PNIC correspondiente al año 2010 fue de aproximadamente US\$726,040.00.

\section{Investigación y Educación}

La primera expedición de la cual se cuenta con registros a la Isla del Coco fue en 1697 con fines exploratorios y comerciales a cargo del pirata y cronista británico William Dampier (Cortés 2008).

La Isla del Coco posee gran cantidad de estudios realizados por distintas organizaciones, trabajos físicos, químicos, biológicos, legales, meteorológicos, culturales, históricos, de sinergia, económicos, geológicos, arqueológicos, sismológicos entre otros. Se han identificado alrededor de 1300 publicaciones, las cuales se encuentran en el centro de documentación del SINAC. Cabe destacar que, el auspicio para 
muchas de las investigaciones realizadas en el PNIC ha sido parte del esfuerzo realizado por FFEM, GEF, el (PNUD), El Ministerio de Ambiente, Energía y Telecomunicaciones (MINAET), Conservación Internacional (CI), Unesco, Embajada de Japón y embajada de Holanda entre otros. Algunas universidades como la Universidad de Costa Rica (UCR) y la Universidad Nacional también han desarrollado trabajos en el PNIC con fondos nacionales.

Además, el Centro de Investigación en Ciencias del Mar y Limnología (CIMAR, Universidad de Costa Rica) ha generado cerca de 20 artículos con los insumos que han obtenido en sus investigaciones, la mayoría de estos artículos se han publicado en la Revista Biología Tropical de la Universidad de Costa Rica.

La investigación resulta importante para los científicos ya que esta zona constituye un santuario en el que cada día se descubren distintas especies, por ejemplo, un proyecto que está siendo desarrollado por la Universidad Nacional y que inició en el 2010, descubrió cinco especies nuevas de helechos. Este proyecto es relevante porque los helechos representan el $30 \%$ de la flora de la isla y es el grupo que mas endemismo tiene. El componente de Conservación trabaja con base en parcelas de 40 metros X 40 metros, en las cuales se puede obtener información de diferentes sitios a diferentes alturas y en diferentes hábitats. Esta información es vital para establecer si las especies están en peligro de extinción o no, que tan abundantes son y planificar cuales sitios requieren de un status de conservación más fuerte o cuales áreas pueden ser utilizadas más para ecoturismo. Con conocimiento sobre las poblaciones de helechos, los científicos pueden plantear a la administración diferentes niveles de conservación (Alexander Rojas com. pers. 2010).

Dentro de los aportes económicos del PNIC en lo referente a investigación y educación se obtuvo información para proyectos de Cooperación Internacional, aportes directos y otros y proyectos con financiamiento nacional.
En el período 2006-2010, dentro de los proyectos de Cooperación Internacional, se obtuvo fondos con el FFEM, CI, Unesco, Embajada del Japón y Embajada de Holanda por montos cercanos a los US\$1,958,235. Para el mismo período por aportes directos que corresponden a compra y mantenimiento de embarcaciones, ayuda en la compra de repuestos y accesorios para diferentes equipos de trabajo y entrega de insumos y accesorios para guardaparques como uniformes, zapatos y otros como parte del trabajo que coordina FAICO para el ACMIC, se obtuvo US\$302,200.

Para el 2010 los montos para estas partidas fue de US\$334,564 para Cooperación Internacional y US\$78,743 para aportes directos (Fig. 3).

Los proyectos con financiamiento nacional son pocos y la mayoría se desarrollan por parte de las universidades públicas con fondos propios o con fondos del Consejo Nacional de Rectores (CONARE). Para el 2010 se pudo obtener información de dos proyectos cuyos montos ascendían a US\$80,292.37.

\section{Actividades Económicas}

En el PNIC la principal actividad económica es el turismo. De esta actividad dependen otras como los Operadores Turísticos, hoteles, restaurantes y empresas de transporte aéreo y terrestre.

El PNIC recibió varios tipos de visitantes en el periodo 1992-2010 (Fig. 4), dentro de los cuales se destacan: turistas, pescadores, investigadores, voluntarios y funcionarios públicos del SINAC y de otras instituciones públicas. El promedio anual de visitación en el periodo fue de 2710 visitantes de los cuales $43 \%$ (1208) fueron nacionales y 57\% (1592) fueron extranjeros. En los últimos años la visitación extranjera ha tenido mayor participación que la de nacionales (Fig. 4). Como la mayoría de visitantes nacionales son exonerados, la mayor parte de los ingresos provienen de los turistas extranjeros. 


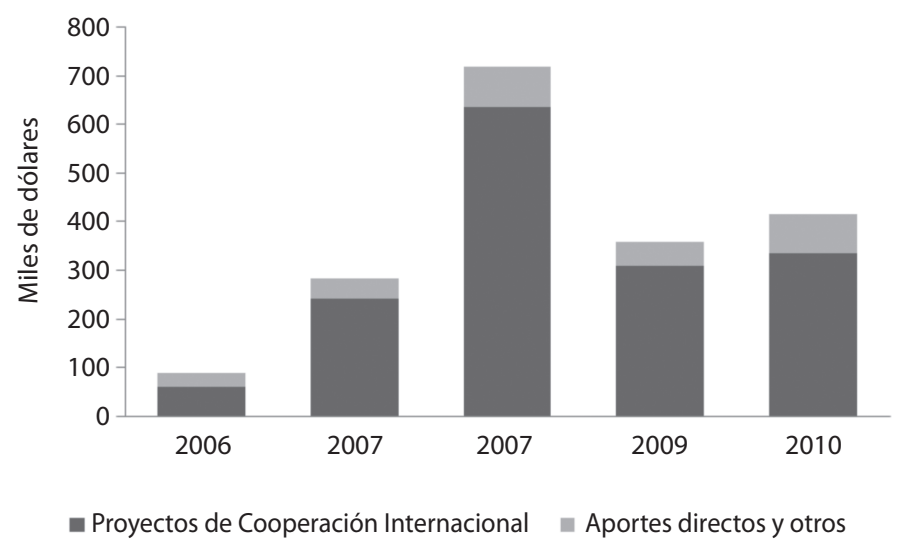

Fig. 3. Detalle estimado de ejecución de proyectos y otros aportes en beneficio del ACMIC. Miles de Dólares, durante el período 2006-2010.Fuente: ACMIC.

Fig. 3. Estimated detail of project execution and other contributions that benefit the ACMIC. Thousands of dollars, during the period 2006-2010. Souce: ACMIC.

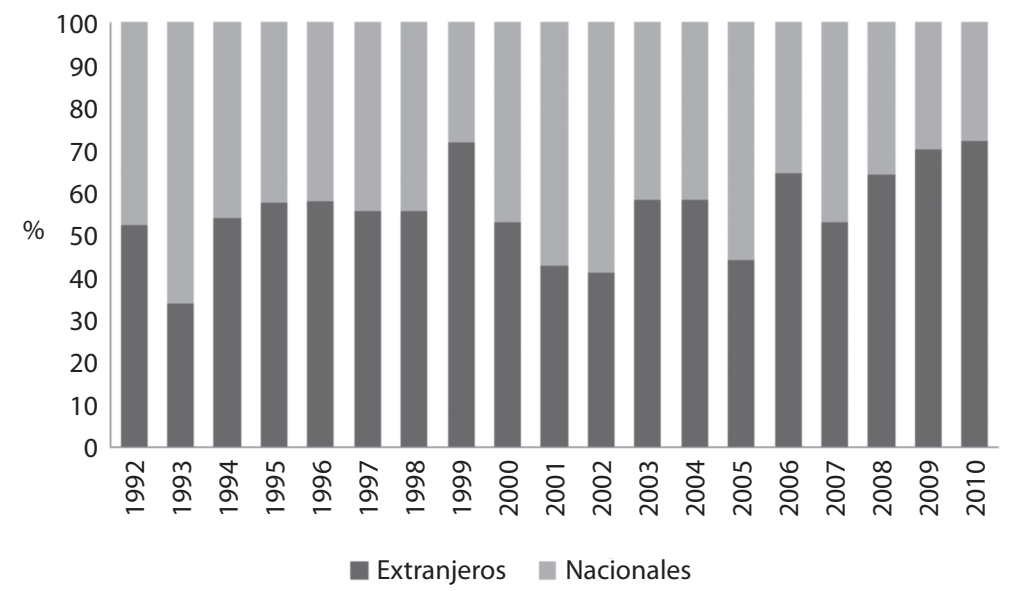

Fig. 4. Visitación Parque Nacional Isla del Coco, 1992-2010 Parque Nacional Isla del Coco. Costa Rica. Fuente: MINAESINAC-ACMIC, 2007 \& SEMEC.2010.

Fig. 4. Visitation to Isla del Coco National Park, 1992-2010.Costa Rica. Source: MINAE-SINAC-ACMIC, 2007 \& SEMEC.2010.

\section{Perfil de visitación y gasto promedio del turista}

Con la información obtenida en el trabajo de campo, se realizó un perfil del turista promedio, considerando los gastos dependiendo de la nacionalidad.

En las encuestas realizadas a los turistas para el presente artículo en el período
2010-2011, se obtuvo que aproximadamente el $74 \%$ de los turistas eran extranjeros mientras que 26\% fueron nacionales. En términos de los países de origen de los turistas, los resultados de las encuestas fueron los siguientes: $26,19 \%$ de Costa Rica, $15,87 \%$ de Italia, $15,08 \%$ de Rusia, $14,29 \%$ de Alemania, 9,52\% de Estados Unidos, 7,14\% de Austria, 2,38\% de México y 2,38\% de Canadá, 2,38\% de Suiza, 
1,59\% de Ucrania, 0,79\% de Francia, España, Polonia y Japón.

De la encuesta se obtuvo también que los turistas realizan de 3 a 4 actividades dentro del Parque Nacional Isla del Coco (Fig. 5). La primera opción es la observación de tiburones (98\%), la segunda opción más seleccionada fue la observación de los arrecifes de corales $(92 \%)$, la tercera fue las caminatas por senderos en el PNIC (65\%) y la cuarta opción fue el snorkeling (31\%). Algunos turistas colocaron otras opciones (17\%).

De la misma manera se destaca que, alrededor del $89,68 \%$ de los encuestados tenían conocimiento sobre la existencia de la Isla del Coco, el 10,32\% restante no respondió. En cuanto a las diferentes formas por las que se enteraron de la existencia del PNIC se identificó que del $89,68 \%$ que conocía sobre la existencia del mismo, el $20,63 \%$ se enteró por amigos, el $15,08 \%$ por vía internet, $15.08 \%$ por medios de telecomunicación, y el restante $49,21 \%$ se enteró por documentales, revistas, estudios, entre otros.

El gasto promedio del turista según nacionalidad para disfrutar del PNIC, que toma en consideración los gastos que realizan los turistas en los tiquetes aéreos, hospedajes, compras en supermercados, alimentación, transporte en taxis, Operadores Turísticos, entrada al PNIC y tarifa diaria de buceo, se presenta en la figura 6 .

\section{Operadores turísticos}

La operación autorizada por el SINAC es realizada por tres empresas de turismo: Aventuras Marítimas Okeanos, Undersea Hunter Group y Golfo Express. La operación se fundamenta en convenios de cooperación entre el MINAET y cada una de las empresas interesadas.

Las empresas cuentan con siete embarcaciones para ofrecer sus servicios. Las dos primeras empresas son las que poseen mayor trayectoria en brindar servicios en el Parque Nacional Isla del Coco. La primera se fundó en 1984, cuenta con dos embarcaciones: Wind Dancer y Okeanos. Tienen la capacidad de brindarle el servicio a 22 pasajeros cada uno, de los cuales aproximadamente el $95 \%$ son extranjeros y el 5\% son costarricenses. Cuentan con una tripulación aproximada de 27 personas. La segunda se crea en 1990, cuenta con 3 embarcaciones: Undersea Hunter, Sea Hunter y Argo, tienen la capacidad de transportar 49 turistas; que en su mayoría son extranjeros $(95 \%$ o más), cuenta con una tripulación de 60 personas en temporada alta y 40 en temporada baja. Adicionalmente es la única empresa que ofrece

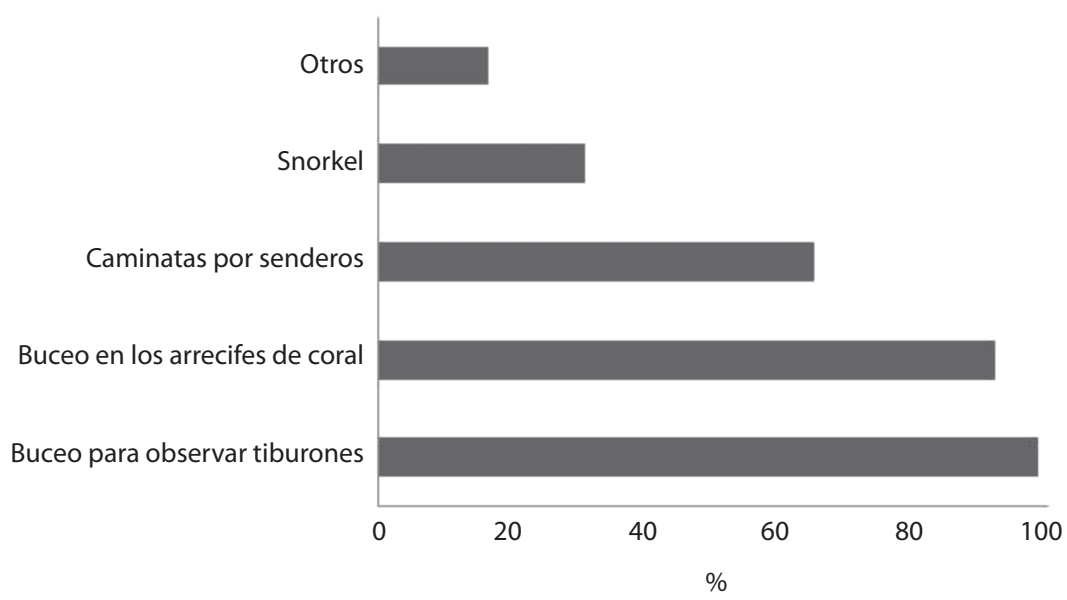

Fig. 5. Porcentaje de visitación según actividad realizada

Fig. 5. Percentage of visitation by activity. 


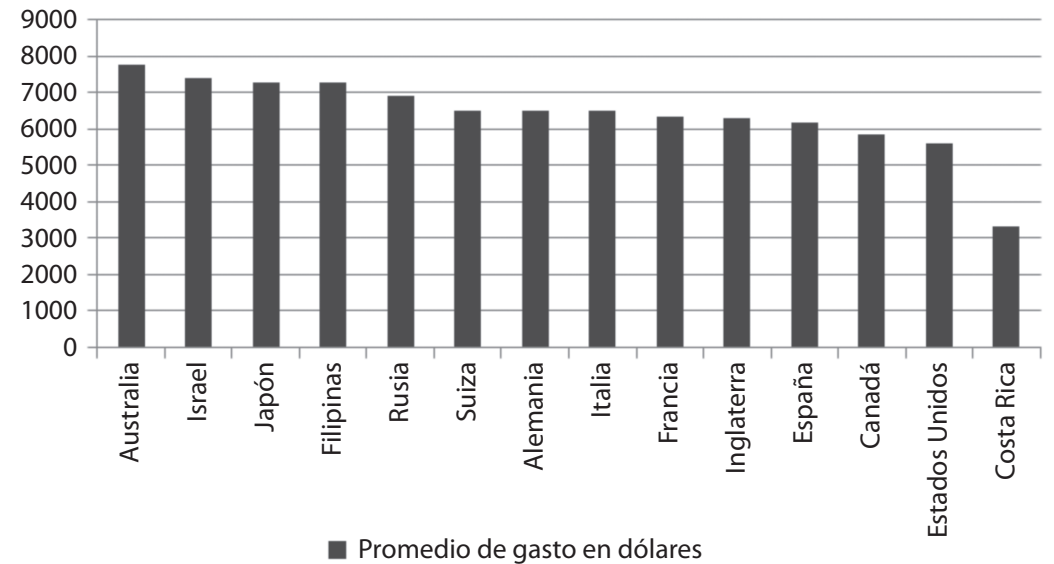

Fig. 6. Gasto promedio del Turista según nacionalidad. 2010.

Fig. 6. Average expenditure of tourist by nationality. 2010.

el servicio del sumergible DeepSee, que ha sido muy importante para la investigación científica.

Por otro lado, Golfo Express fue creada en el 2006 y cuenta con una embarcación; Adventure, la cual tiene capacidad para 18 turistas (aproximadamente el 99\% son extranjeros y $1 \%$ nacionales). Cuenta con una tripulación de 15 personas en temporada alta y 6 en temporada baja.

Estas empresas se especializan en el transporte de turistas por lo cual el $100 \%$ de sus ingresos provienen de los mismos. Los paquetes que adquieren los turistas extranjeros y nacionales de las distintas empresas oscilan entre 10 y 12 días y su precio varía de acuerdo a la temporada y a la embarcación que elija el turista. Los Operadores Turísticos se especializan en la actividad del buceo.

En el trabajo de campo se entrevistó el siguiente número de turistas en cada embarcación: Okeanos 22, Sean Hunter 25, Under Sean Hunter 24, Pacific Explorer 34, Golfo Express 9, Wind Dancer 12.

El PNIC también es visitado por pequeños navíos que transportan pocos visitantes y que permanecen en la isla menos de 10 días. En 2007 se contabilizaron 40 embarcaciones de este tipo que transportaron 335 visitantes.

\section{Aportes económicos de los Operadores Turísticos}

Para el año 2010, los ingresos totales de los Operadores Turísticos que llevan turistas al PNIC fue de aproximadamente $\$ 3,761,700$. Este monto fue distribuido entre varias actividades que prestan sus servicios a las empresas (Fig. 7), el mayor gasto se produce para la compra de combustible, luego viene otros gastos y utilidades, gastos de mantenimiento, alimentos, salarios, bebidas, servicios básicos (electricidad, agua) y otros servicios (internet, limpieza y televisión por cable).

Transporte. Al llegar a Costa Rica, los turistas deben tomar un taxi desde el aeropuerto a los hoteles recomendados. Al día siguiente toman una buseta que los lleva a Puntarenas, donde se encuentran los muelles de los Operadores Turísticos. El costo del transporte en la buseta es incluido en el paquete todo incluido que ofrece el Tour Operador. Aproximadamente el $10 \%$ de los turistas no utiliza el servicio, ya que, prefiere realizar tours antes o después de ir al PNIC y por lo tanto contrata su propio medio de transporte. 


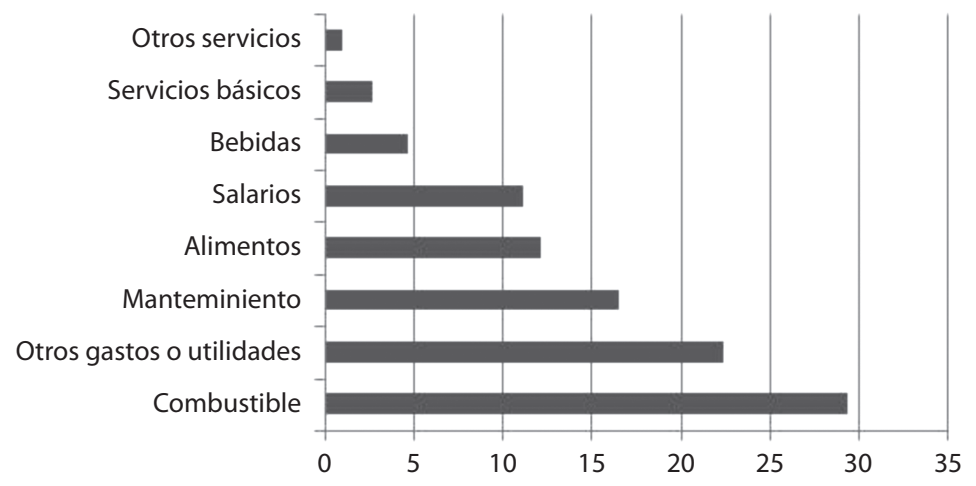

Fig. 7. Distribución del ingreso de los Operadores Turísticos a otras actividades productivas. (\%).

Fig. 7. Income distribution of Tour Operators to other productive activities (\%).

Algunos de los Operadores Turísticos establecen convenios con las empresas de transporte y cada una de ellas realiza en promedio nueve viajes mensuales, estas busetas tienen una capacidad promedio de 30 personas cada una y también se contrata un camión para el trasporte del equipo de buceo de los turistas.

Hoteles. Algunos Operadores Turísticos recomiendan al visitante pasar dos noches en la capital (San José); un día antes de dirigirse al PNIC y un día después.

Aproximadamente el $90 \%$ de los turistas se hospedan en seis hoteles recomendados por algunos de los Tour Operadores, lo que en promedio indica que un total de 896 personas utilizan este tipo de servicio. El gasto total por persona es de US\$522 por los dos días por hospedaje y alimentación. Es decir los hoteles percibieron por concepto de hospedaje de los turistas que se dirigen al PNIC en el 2010, un monto aproximado de US\$467,712.00.

Comercios y Supermercado. En su trayecto hacia Puntarenas, los turistas que se dirigen al PNIC, emplean los servicios de un restaurante, que de acuerdo a la información obtenida en el trabajo de campo, atiende a cerca de 476 de estos turistas al año, con un ingreso anual de US\$2,856.

Algunos de los turistas compran en Puntarenas en varios comercios pequeños y medianos como supermercados y pulperías. El gasto promedio es de $\$ 20$.

\section{Aportes del PNIC en el ámbito local, nacional e internacional}

Las actividades descritas anteriormente generan ingresos a nivel local, nacional e internacional. En esta sección se separaron estos ingresos y se presentan en el Cuadro 1. Tal como se muestra en las secciones anteriores, en el PNIC operan una serie organizaciones, personas y empresas que generan interacciones con otros actores y brindan aportes económicos en el ámbito local (Puntarenas), nacional y en el internacional. En la presente investigación se pudo obtener datos para los ámbitos nacional e internacional pero no para el local. En este sentido es importante reiterar que los montos aquí presentados son una aproximación de los montos reales y que debido a la gran dificultad para conseguir este tipo de información probablemente estén subvalorados.

En el Cuadro 1 se muestra el detalle de los aportes en el ámbito nacional e internacionales del PNIC, que para el 2010 fue de US $\$ 8,342,245.25$.

La mayor contribución del PNIC en la generación de ingreso se presentó para las actividades económicas $(50.83 \%)$, posteriormente se destaca el ingreso generado para aerolíneas que transportan a los turistas desde diferentes 
CUADRO 1

Sistematización de los Ingresos Brutos obtenidos por las actividades económicas en el PNIC. 2010

TABLE 1

Systematization of the gross income derived from economic activities in the PNIC. 2010

\begin{tabular}{|c|c|c|}
\hline $\begin{array}{c}\text { Alcance-escala } \\
\text { del aporte }\end{array}$ & Actividades beneficiadas. Tipo de Actividad & $\begin{array}{c}\text { Tipo de aporte valorado } \\
\text { según fuentes (Ingresos) } \\
\text { (Dolares) }\end{array}$ \\
\hline \multirow[t]{24}{*}{ Nacional } & Gestión del PNIC (12,67\%) & $1056.251,26$ \\
\hline & Entradas a los Parques Nacionales & $586.582,58$ \\
\hline & Servicios varios & $30.913,27$ \\
\hline & Salarios (con Fondos SINAC) & $299.297,99$ \\
\hline & Salarios (con dineros del Fondo Nacional de P) & $139.457,42$ \\
\hline & Investigación y Educación (5,88\%) & $493.599,37$ \\
\hline & Proyectos de Cooperación Intemacional & $334.564,00$ \\
\hline & Aportes directos y otros & $78.743,00$ \\
\hline & Proyectos con fondos nacionales & $80.292,37$ \\
\hline & Actividades Económicas e Insumos (50,83\%) & $4.238 .793,72$ \\
\hline & Combustible & $1103.565,96$ \\
\hline & Matenimiento & 621293,88 \\
\hline & Alimentación turistas & $457.201,68$ \\
\hline & Salarios & $418.497,48$ \\
\hline & Bebidas & $174.750,60$ \\
\hline & Servicios básicos & $100.216,20$ \\
\hline & Internet & $4.264,80$ \\
\hline & Limpieza & $7.581,00$ \\
\hline & Televisión por cable & 1453,68 \\
\hline & Otros Servicios & $36.508,44$ \\
\hline & Otros Gastos o Utilidades & $842.892,00$ \\
\hline & Servicios de Hotelería & $467.712,00$ \\
\hline & Servicios de Restaurante & $2.856,00$ \\
\hline & Total Nacional & $5.788 .644,35$ \\
\hline \multirow[t]{2}{*}{ Internacional } & Aerolineas $(30,62 \%)$ & $2.553 .600,90$ \\
\hline & Total Internacional & $2.553 .600,90$ \\
\hline Total Ingresos & & $8.342 .245,25$ \\
\hline
\end{tabular}

países $(30.62 \%)$, los ingreso generados por la gestión del Parque (12.67\%) y por último los fondos generados para investigación y educación $(5.88 \%)$. Si se analizan los resultados de acuerdo a la escala, se obtiene que los mayores ingresos se produjeron en el ámbito nacional $(69.38 \%)$ y luego en el internacional (30.62\%). En el ámbito local no se calcularon los ingresos por la dificultad en la consecución de la información, sin embargo se puede afirmar que estos ingresos sí existen.

\section{Posibles efectos de la Variabilidad \\ Climática sobre los ingresos de las actividades socioeconómicas}

Con base en resultados obtenidos por Sibaja-Cordero (2008), en donde se concluye que el Fenómeno del Niño ha tenido repercusiones sobre la abundancia y presencia de especies como la del tiburón martillo, se les consultó a los turistas si ellos seguirían visitando la Isla del Coco aunque la especie objeto de su visita 


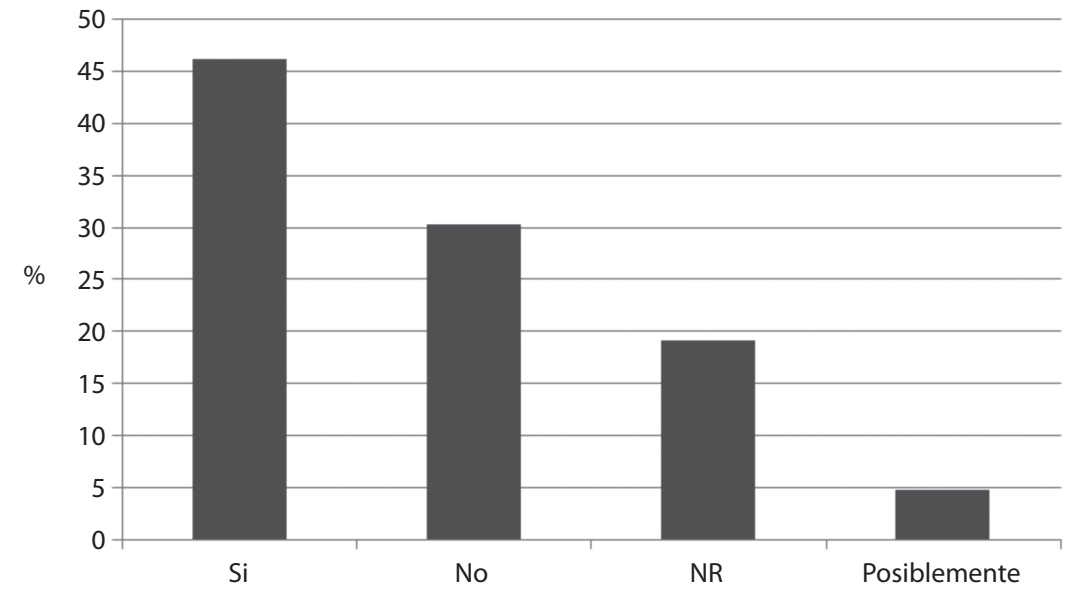

Fig. 8. Comportamiento de los turistas ante la desaparición del recurso natural por el que visitan el PNIC. Fig. 8. Behavior of tourists to the disappearance of the natural resource of their interest in PNIC.

disminuyera o desapareciera, el 46\% manifestó que si regresaría, el $30 \%$ que no, el $24 \%$ no respondió y el $6 \%$ restante posiblemente regresaría (Fig. 8).

Empleando la metodología de Fürst (2004), se realiza el supuesto de que la reducción del $30 \%$ en la visitación por desaparición o disminución de los recursos marinos traerá como consecuencia una disminución en el mismo porcentaje de los aportes generados por esta visitación. Con base en los resultados obtenidos para el 2010 y presentados en el Cuadro 1, esta disminución seria de US\$2,502,637.57.

\section{DISCUSIÓN}

Las principales actividades socioeconómicas que se desarrollan alrededor de la existencia del PNIC son el manejo y la gestión, la investigación y la educación y algunas actividades económicas. Estas actividades giran en torno a la conservación de los recursos naturales del Parque y a la actividad turística que se desarrolla en él.

Estos resultados aunque sin el detalle presentado aquí, se han obtenido para otras Áreas Marinas Protegidas (AMP), las cuales impactan positivamente la biodiversidad marina y la recuperación de especies para la pesca, además de proporcionar importantes espacios para actividades como el turismo (Otoya et al. 2010). Lo anterior debido a que al incrementar las densidades de peces dentro de las AMP, las capturas fuera de estas áreas probablemente aumentarán (Ruijs \& Janmaat 2007) y a que estas áreas permiten la conservación y protección de especies que son objeto de las actividades turísticas. En este sentido una contribución importante de las AMP, es que permiten que, poblaciones de especies objetivo sean más estables que cuando se toman medidas de recuperación fuera de ellas (Babcock et al. 2010). En este sentido el PNIC, proporciona aportes al país no solamente por la protección en recursos marinos importantes como el tiburón martillo y la raya moteada sino que diferentes actividades económicas y sociales se desarrollan debido a su existencia.

Se demostró en este articulo que la aplicación de la metodología conglomerado-cadena para parques nacionales y reservas biológicas creada por Fürst et al. (2004), puede ser empleada en el caso de AMP también. Esta metodología permitió cuantificar los múltiples efectos de vinculación hacia delante, que tiene el PNIC en términos socioeconómicos. Los resultados obtenidos aunque en términos numéricos se diferencia de otros estudios (Fürst 
et al. 2004, Naranjo-Barrantes 2007, Moreno et al. 2010, Otoya et al. 2010, Salas et al. 2010), permiten obtener conclusiones similares en cuanto a la importancia de los parques nacionales a la economía nacional y a que esta importancia se debe identificar a diferentes niveles. Como en estos estudios, en el caso del PNIC, las actividades económicas son las que más ingresos obtienen de su existencia, aunque también se generan importantes contribuciones en el manejo y gestión y en la investigación y la educación.

Aunque se ha tratado de identificar y sistematizar los aportes más relevantes del PNIC al desarrollo socioeconómico, bajo la perspectiva que diferencia entre las escalas espacialesterritoriales del lugar (Parque Nacional), zona o región (Área de Conservación ) y país (institucionalidad), la escala a nivel nacional ha prevalecido con respecto a los datos y resultados encontrados. Un resultado parecido se encontró en los estudios relacionados y mencionados en el párrafo anterior. En el caso del presente estudio, los ingresos a nivel local (Puntarenas) no pudieron ser cuantificados debido a la dificultad en la consecución de la información, pero definitivamente existen. Un resultado interesante que también se dio en el estudio de Salas et al. (2010), es que los ingresos que obtienen diferentes empresas a nivel internacional de la existencia del PNIC son importantes y en general no se consideran como posibles contribuyentes para el mejoramiento de esta área.

Por otro lado los posibles efectos de la variabilidad climática identificados sobre los recursos naturales del Parque (Sibaja-Cordero 2008), es la disminución en la abundancia de especies importantes para el turismo como el Tiburón Martillo, esto podría conllevar disminuciones de hasta $30 \%$ en los ingresos obtenidos de la existencia del PNIC, debido a la disminución de turistas.

Una reflexión final es que a pesar de la gran importancia socioeconómica y el ingreso que muchas de las AMP generan a las actividades socioeconómicas que se desarrollan alrededor de ellas, algunas presentan problemas en su financiamiento y el PNIC no es la excepción.
Una posible causa es la relación entre visitantes y requerimiento de ingreso (Gravestock et al. 2010). Recientemente se han desarrollado esfuerzos en torno a la posibilidad de crear redes de AMP para mejorar la conservación de éstas y potenciar sus ingresos y beneficios para la actividad turística y pesquera generada fuera de ellas (Costello et al. 2010, Gaines et al. 2010). En el caso del PNIC la iniciativa del Corredor Marino de Conservación del Pacífico Este Tropical, tiene entre sus objetivos el impulso de la actividad turística responsable y brindar un conjunto de bienes y servicios ambientales a escala local, regional y nacional (Rodríguez 2005, McCook et al. 2010 ).

\section{AGRADECIMIENTOS}

A los empresarios, investigadores, funcionarios del SINAC-ACMIC y de otras instituciones públicas y privadas por su disponibilidad y valiosa colaboración en la consecución de la información. A Daniela Murillo Ruiz y Jackeline Cruz Chaves por el apoyo en la consecución y sistematización de la información de campo. Este trabajo se realizó gracias al apoyo del proyecto "Interacciones océano-atmósfera y la biodiversidad marina de la Isla del Coco, Costa Rica" financiado por el Consejo Nacional de Rectores (CONARE), Costa Rica. Este trabajo es parte del trabajo doctoral de la autora dentro del Doctorado en Ciencias Naturales para el Desarrollo (DOCINADE), programa conjunto de la Universidad Nacional (UNA), Costa Rica, Instituto Tecnológico de Costa Rica (TEC) y la Universidad Estatal a Distancia (UNED), Costa Rica.

\section{RESUMEN}

Las actividades socioeconómicas y sus ingresos en el Parque Nacional Isla del Coco han sido poco evaluados y sistematizados, al igual que los efectos que sobre ellos pueda tener la variabilidad climática. empleó una metodología que combina análisis de conglomerados con una dinámica de valor agregado en una cadena productiva, para identificar las actividades socioeconómicas más importantes y cuantificar los ingresos que obtienen por la existencia del PNIC, además se analizó los posibles efectos 
de la variabilidad climática sobre estas actividades. Para lo anterior, se realizó una revisión bibliográfica detallada, consulta con empresarios, investigadores y turistas. Se obtuvo que los principales conglomerados de actividades alrededor del PNIC son la recreación y la vivencia espiritual, la investigación, las actividades económicas y la gestión y manejo del Parque. Se realizó una aproximación de las contribuciones de la existencia del PNIC en estos cuatro conglomerados en el 2010, y se obtuvo que la contribución el ámbito nacional fue de US\$5.7 millones de dólares, el internacional de US\$2.5 millones de dólares, el global del PNIC ascendió aproximadamente a US\$8.3 millones de dólares. También se obtuvo que si los recursos naturales objeto de la visita de los turistas disminuyen debido a efectos de la variabilidad climática esto ingresos podrían disminuir en $30 \%$.

Palabras clave: Parque Nacional Isla del Coco, ingresos, beneficios socioeconómicos, conglomerado, variabilidad climática, valor agregado, Costa Rica.

\section{REFERENCIAS}

Alfaro, E.J. 2008. Ciclo diario y anual de variables troposféricas y oceánicas en la Isla del Coco, Costa Rica. Rev. Biol. Trop. 56 (Supl. 2): 19-29.

Altenburg, T. \& J. Meyer-Stahmer. 1999. How to Promote Clusters: Policy Experiences from Latin America. World Develop. 27: 1693-1713.

Alvarado, J.J., B. Herrera, L. Corrales, J. Asch \& P. Paaby. 2011. Identificación de las prioridades de conservación de la biodiversidad marina y costera en Costa Rica. Rev. Biol. Trop. 59: 829-842.

Babcock, R.C., N.T. Shears, A.C. Alcala, N.S. Barrett, G.J. Edgar, K.D. Lafferty \& G.R. Russ. 2010. Decadal trends in marine reserves reveal differential rates of change in direct and indirect effects. Proc. Nat. Acad. Sci. USA 107: 18256-18261.

Bermúdez-Acuña, F., I. Zanella \& M. Ballestero. 2007. Plan de uso público del Parque Nacional Isla del Coco. Onca Natural/MINAE/SINAC, San José, Costa Rica. $60 \mathrm{p}$.

Cajiao, M.V. 2008. Aspectos legales relacionados con el Parque Nacional Isla del Coco, Costa Rica. Rev. Biol. Trop. 56 (Supl. 2): 207-214.

Chae D, P. Wattage \& S. Pascoe. 2012. Recreational benefits from a marine protected area: A travel cost analysis of Lundy. Tourism Management. Volume 33. 4: 971-977

Cortés, J. 2008. Historia de la investigación marina de la Isla del Coco, Costa Rica. Rev. Biol. Trop. 56 (Supl. 2): $1-18$.

Costello, C., A. Rassweiler, D. Siegel, G. De Leo, F. Micheli \& A. Rosenberg. 2010. The value of spatial information in MPA network design. Proc. Nat. Acad. Sci. USA 107: 18294-18299.

Del Saz, S\& C. Suárez. 1998. El valor de uso recreativo de espacios naturales protegidos: aplicación del método de valoración contingente al Parque Natural de L'Albufera. Economía Agraria. 182: 239-272.

Dixon J., Fallon L., Carpenter R., Sherman P., 1994. Análisis Económico de Impactos Ambientales. Earthhscan Publications, Ltd.

Fürst, E., M.L. Moreno, D. García \& E. Zamora. 2004. Desarrollo y conservación en interacción: ¿cómo y en cuánto se benefician la economía y la comunidad de las áreas silvestres protegidas en Costa Rica? INBio \& CINPE, Heredia, Costa Rica. (Consultado: 11 octubre 2011, www.inbio.ac.cr/otus/pdf/PNRB.pdf).

Freeman, A. III. 1993. The measurement of Environmental and Resources Values. Theory and Methods. Resources for the Future. Washington 516p.

Gaines, S.D., C. White, M.H. Carr \& S.R. Palumbi. 2010. Designing marine reserve networks for both conservation and fisheries management. Proc. Nat. Acad. Sci. USA 107: 18286-18293.

Garrod G., Willis K. 1999. Economic Valuation of the Environment. Methods and Case Studies. Cheltenham: UK and Northampton, MA, USA: Edward Elgar.

Gravestock, P., C.M. Roberts \& A. Bailey. 2007. The income requirements of marine protected areas. Ocean Coast.Manag. 51: 272-283.

McCook, L.J., T. Ayling, M. Cappo, J.H. Choat, R.D. Evans, D.M. De Freitas, M. Heupel, T.P. Hughes, G.P. Jones, B. Mapstone, H. Marsh, M. Mills, F.J. Molloy, C.R. Pitcher, R.L. Pressey, G.R. Russ, S. Sutton, H. Sweatman, R. Tobin, D.R. Wachenfeld \& D.H. Williamson. 2010. Adaptive management of the Great Barrier Reef: A globally significant demonstration of the benefits of networks of marine reserves. Proc. Nat. Acad. Sci. USA 107: 18278-18285.

MINAE-SINAC-ACMIC. 2007a. Plan de Uso Público del Parque Nacional Isla del Coco. San José, Costa Rica.

MINAE-SINAC-ACMIC. 2007b. Plan de Manejo del Parque Nacional Isla del Coco. Documento Técnico. San José, Costa Rica. 122 pp. (Consultado: 15 de marzo 2011, http:/www.isladelcoco.go.cr/attachments/079 Plan\%20de\%20Manejo\%20PNIC.pdf)

Moreno, M.L., S. González \& C. Mora. 2010. Analysis of the Socio-economic Contributions of Palo Verde National Park. "A Nest for Research and Education" 2009. Documento Técnico. UNA, CINPE, SINAC, Heredia, Costa Rica.

Moreno, M.L., S. Choden, A. Floquet \& R.L. Mongbo. 2011. Protected Areas-not Just for Biodiversity Conservation: The Contributions ofProtected Areas to the Economic and Social Development in Bhutan, Costa 
Rica and Benin. Edit. Zeta Servicios Gráficos S.A., San José, Costa Rica.

Naranjo-Barrantes, A. 2007. The contribution of protected nature areas towards socio-economic development in Costa Rica: A cluster analysis of Braulio Carri1lo National Park. Wageningen Univ., Gelderland, Holanda.

Obando, M. 2005. Microcentral hidroeléctrica Genio Parque Nacional Isla del Coco, Costa Rica. Instituto Costarricense de Electricidad (.ICE). Informe Ejecutivo. San José, Costa Rica. 22p.

Otoya, M., M.L. Moreno, D. Cordero \& C. Mora. 2010. Analysis of the Socio-economic Contributions of Corcovado National Park and Caño Island Biological Reserve. "The Biological, Archaeological, Cultural Richness of the South Coast Mediated by the Dynamism of its Villages". Documento Técnico. UNA, CINPE, SINAC, Heredia, Costa Rica.

Pearce, D. \& K. Turner. 1995. Economía de los recursos naturales y del medio ambiente. Edit. Alianza, Madrid, España.

Porter, M. 1990. The Competitive Advantage of Nations. Edit. Simon \& Schustre, Nueva York, Estados Unidos.

Porter, M. 1999. Clusters and the New Economics of Competition. Documento 10 presentado al taller de trabajo sobre "Conceptos y Metodologías en el análisis de clusters". CEPAL, Santiago, Chile.

Quirós-Badilla, E. \& E.J. Alfaro. 2009. Algunos aspectos relacionados con la Variabilidad Climática en la Isla del Coco, Costa Rica. Rev. Climatol. 9: 33-44.
Ramos, J. 1999. Una estrategia de desarrollo a partir de los complejos productivos (clusters) en torno a recursos naturales. ¿Una estrategia prometedora? Documento 1 presentado al taller de trabajo sobre "Conceptos y Metodologías en el análisis de clusters". CEPAL, Santiago, Chile.

Rodríguez, J. 2005. Historia y carácter del Corredor Marino. Rev. Ambientico 138: 6-8.

Ruijs, A. \& J.A. Janmaat. 2007. Chasing the spillovers: Locating Protected Areas in a trans-boundary fishery. Land Econom. 83: 6-22.

Salas F., M.L. Moreno, S. González \& C. Mora. 2010. Analysis of the Socio-economic Contributions of Rincón de la Vieja National Park "Conservation with Soul of Volcano" World Natural Heritage Site UNESCO".Documento Técnico. UNA, CINPE, SINAC, Heredia, Costa Rica.

Scitowsky, T. 1973. Dos conceptos de las economías externas, pp. 248-258. In A.N. Agarwala \& S.P. Singh (eds.). La Economía del Desarrollo. Edit. Tecnos, Madrid, España.

Sibaja-Cordero, J.A. 2008. Tendencias espacio-temporales de los avistamientos de fauna marina en los buceos turísticos (Isla del Coco, Costa Rica). Rev. Biol. Trop. 56 (Supl. 2): 113-132.

Wang, P. \& J. B. Jia. 2012. Tourists' willingness to pay for biodiversity conservation and environment protection, Dalai Lake protected area: Implications for entrance fee and sustainable management. Ocean and Coastal Management. Volume 62: 24-33. 
